\title{
La labor tutorial y su impacto en la formación científico-investigativa inicial del docente
}

\author{
Diane González Mirabal ${ }^{1}$ \\ Gustavo Esteban Achiong Caballero ${ }^{1}$
}

\section{Resumen}

Este artículo presenta los resultados de una investigación de doctorado que estudió el proceso de tutoría en la formación científico-investigativa inicial del profesional de la educación en Cuba, teniendo en cuenta que la investigación científica constituye uno de los componentes esenciales y contenido de una de las funciones que desempeña el docente una vez graduado. En este escenario, la tutoría personalizada desempeña un importante papel ya que debe garantizar, a partir de la atención de las necesidades y potencialidades del estudiante tutorado, el desarrollo y sistematización del sistema de conocimientos y habilidades investigativas que le propicie desplegarlas en la realización de su trabajo científico-investigativo. En respuesta a esta necesidad se presenta una metodología que contribuya al perfeccionamiento de la tutoría en la formación científico-investigativa inicial del profesional de la educación. En la investigación se utilizaron métodos del nivel teórico, empírico y estadístico. La significación práctica se concreta en los procedimientos para la proyección y ejecución de la labor tutorial. La evaluación de la metodología se realizó mediante la aplicación del criterio de expertos y el análisis de los datos obtenidos durante el desarrollo de un pre-experimento pedagógico. Los resultados permiten afirmar que se logra efectividad en la acción tutorial con la aplicación de la metodología propuesta, posibilitando al profesor-tutor conducir al estudiante a través de una relación de ayuda que orienta su desarrollo y apoya sus necesidades en la actividad científico-investigativa, de forma que contribuye a la sistematización de sus habilidades en esta dirección.

\section{Palabras clave}

Formación científico-investigativa - Tutoría en la formación científico-investigativa Acción tutorial - Niveles de ayuda en la tutoría.

1- Universidad de Sancti Spíritus, Sancti Spíritus, Cuba.

Contactos: dgmirabal@uniss.edu.cu; gachiong@uniss.edu.cu

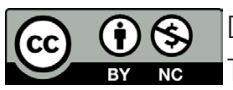




\section{Tutorial work and its impact in professor's research scientific formation}

\section{Abstract}

This article presents the results of a doctorate research that studied the tutoring process in the initial scientific research formation of education professionals in Cuba, taking into account that scientific research constitutes one of the essential components and content of one of the functions carried out by teachers after graduation. In this scenario, personalized tutoring plays an important role as it should guarantee, beginning with the attention to the needs and potentialities of the tutored student, the development and systematization of the system of knowledge and research abilities to be utilized when carrying out the scientific research work. Responding to this necessity, a methodology that contributes to improving tutoring in the initial scientific research formation of education professionals is presented. Methods of theoretical, empirical and statistical levels were used. The practical significance of this research lies on the procedures for the projection and execution of the tutorial work. The evaluation of the methodology was carried out through the application of experts' criteria and the analysis of the data obtained from the development of a pedagogical pre-experiment. Results allow to assert that effectiveness in tutorial action with the application of the proposed methodology is achieved, allowing the tutor professor to guide student through a helping relationship that guides the student's development and supports his or her needs in scientific research activity, so that it contributes to the systematization of the student's abilities in this direction.

\section{Keywords}

Research scientific formation - Tutoring in the research scientific formation - Tutorial action - Helping levels in tutoring.

\section{Introducción}

En la actualidad, un propósito fundamental del proceso de formación inicial del profesional de la educación en Cuba es lograr la calidad y rigor que se requiere, como respuesta a las políticas económica y social trazadas por el país. En correspondencia con este objetivo se exige una concepción y práctica renovadoras, se potencia la flexibilidad del currículo y el perfeccionamiento continuo.

En este sentido se consolida en dicho proceso la interrelación entre los componentes académico, laboral, investigativo y extensionista; profundizando en los requerimientos que demanda la sociedad que exige profesionales preparados, motivados, flexibles, que reconozcan la necesidad de solucionar los problemas que en su realidad educativa enfrentan. 
Existe consenso en la actualidad al plantearse que no es posible hablar de calidad de la educación sin investigación. Esta constituye una de las funciones que el docente desarrolla para el perfeccionamiento del proceso pedagógico, utilizando métodos propios de la investigación en la solución de problemas científicos en estrecha interrelación e interdependencia con las funciones docente-metodológica y orientadora.

Es por ello que la formación científico-investigativa inicial es esencial, en tanto le permite al futuro profesional problematizar, indagar, reflexionar acerca de la realidad educativa y lograr transformaciones tanto en el contexto de actuación como en un plano individual.

Mediante la actividad científico-investigativa el estudiante se apropia del método científico a partir de la adquisición de conocimientos, del desarrollo de hábitos y habilidades investigativas que le permite utilizar de forma creadora los conocimientos en la teoría y en la práctica para dar solución, por la vía científica, a los problemas que enfrenta.

Las aspiraciones se centran en la formación de un maestro investigador de su propia práctica educativa a partir de la utilización de métodos y formas de trabajo habituales en la actividad científica para resolver los problemas que se presentan en su contexto de actuación.

En la literatura científica se destacan los estudios realizados por autores extranjeros como Stenhousen (1987), Porlán (1987), Tonucci (1988) y Fernández Muñoz (1995) entre otros, que han sustentado la necesidad de la formación del maestro investigador, como elemento de cambio que influye decisivamente en la calidad de los procesos educativos.

Asimismo, destacados investigadores cubanos han presentado valiosos estudios en relación con la formación científico-investigativa del profesional de forma general, entre los que se pueden citar: Castellanos (1998), López (2001), Chirino (2002), García Batista y Addine Fernández (2004), Salazar (2004) y Pérez (2005). Sin embargo, de forma general prevalecen las concepciones que estos poseen acerca del proceso investigativo desde el punto de vista de su lógica instrumental, sobre aquellas que deben responder a las particularidades del proceso de enseñanza-aprendizaje.

Con el propósito de cumplir con la aspiración de formar a un maestro investigador de su propia práctica, en el currículo base de los Planes del Proceso Docente (PPD) vigentes para las carreras pedagógicas se establecen disciplinas que desempeñan un importante papel pues fomentan la búsqueda del conocimiento, el desarrollo del pensamiento científico y de las habilidades científico-investigativas en el estudiante, que son la base para encontrar soluciones a los problemas profesionales garantizando el dominio de los modos de actuación esenciales de la profesión.

La influencia coordinada de todos los agentes formativos debe contribuir de forma general a la formación integral del futuro profesional de la educación y de forma particular a su formación científico-investigativa, en la que la labor del tutor es esencial.

En los estudios realizados en esta investigación se identifica la contradicción existente entre las limitaciones de los procederes que en la actualidad caracterizan la tutoría en la formación científico-investigativa inicial del profesional de la educación y las exigencias que, en el modelo de formación profesional pedagógico, se plantean a la tutoría para la conducción personalizada de dicha formación en los estudiantes. En consecuencia, se formuló el siguiente problema científico: ¿Cómo contribuir a perfeccionar la tutoría en la formación científico-investigativa inicial del profesional de la educación 
en la Universidad de Sancti Spíritus? y como objetivo proponer una metodología para la tutoría en esta dirección que contribuya al perfeccionamiento de dicho proceso.

Se comprobó la factibilidad y rigor científico de la metodología propuesta, sobre la base de la aplicación del método criterio de expertos y el empleo del pre-experimento para medir la efectividad de la acción tutorial en la formación científico-investigativa del estudiante, con la constatación realizada en la práctica pedagógica.

\section{Marco teórico}

\section{Tendencias de la formación científico-investigativa inicial del profesional de la educación en Cuba}

En la actualidad se sitúa a la educación como un factor y medio importante que influye en el desarrollo y en la calidad de vida del hombre, teniendo en cuenta que como proceso y resultado lo prepara para la vida en todas sus facetas, tanto individual como en su interacción y desempeño social.

Tal es así, que bajo el impacto que ha provocado el vertiginoso desarrollo de la ciencia y la tecnología alcanzado en los inicios del siglo XXI en el mundo del conocimiento y la información, resulta predominante en la actualidad la concepción de la educación para toda la vida, reconocida bajo el término de educación permanente, lo cual ha derivado a su vez, en la consideración de que la calidad y el perfeccionamiento de los procesos pedagógicos sean también una constante en todos los subsistemas educativos.

En consecuencia, la universidad cubana desarrolla planes de mejora en busca de la excelencia en la calidad de sus procesos, donde la investigación ocupa un lugar significativo por ser un elemento esencial en la formación del profesional, constituyendo uno de los parámetros a considerar en los procesos de acreditación y reconocimiento de alta calidad de las instituciones de Educación Superior.

$\mathrm{Al}$ respecto la Organización para la Educación, la Ciencia y la Cultura de las Naciones Unidas en la Conferencia Mundial sobre la Educación Superior, precisa entre las misiones y funciones de dichas instituciones en el siglo XXI, en el artículo I, "la misión de educar, formar y realizar investigaciones" (UNESCO, 1998, p. 4).

Por lo que en efecto, formar el profesional que se necesita exige de la universidad enseñar a pensar y a investigar en y desde la práctica educativa. Esta tendencia, muy fortalecida en la actualidad tiene su sustento en la concepción de formar un maestro investigador, fundamentada desde diferentes aristas por autores extranjeros como Stenhousen (1987), Porlán (1987) y Tonucci (1988) y más recientemente por pedagogos cubanos como López, Pérez y Cáceres (2004), Pérez (2005), Laguna y Sánchez (2005) entre otros, que destacan la importancia de la investigación en el desempeño del docente como elemento de cambio y de mejora educativa.

Esta concepción surge y se desarrolla en los diferentes países desde la década del sesenta del pasado siglo. Se le atribuye a Lawrence Stenhousen el origen de esta expresión, quien al elaborar un nuevo enfoque curricular en la escuela concibe el modelo de profesor investigador en el aula. En torno a este propósito se sumaron destacados pedagogos en 
Francia, Italia, España, los que reconocieron el lugar que ocupa la investigación en la escuela y en particular el papel que en ella desempeña el maestro.

La formación del maestro investigador constituye una problemática actual debido a la necesidad de que los propios maestros construyan la teoría de su práctica pedagógica atendiendo a las características del proceso pedagógico en que intervienen y a las exigencias actuales que se les plantean a las instituciones educativas.

No obstante, existen diversas posiciones en cuanto a la relación docenciainvestigación que van desde planteamientos que afirman que todo docente es investigador hasta aquellos que defienden la imposibilidad de combinarla. Dichos planteamientos tienen su reflejo en el currículo de la formación del docente. De forma general, en la actualidad se reconoce que la presencia de la investigación es uno de los elementos definitorios de los centros de calidad.

Los estudios llevados a cabo por la Oficina Regional de Educación de la UNESCO para América Latina y El Caribe (OREALC; UNESCO, 2006), en los que se analizaron siete modelos de formación inicial de docentes de centros académicos de América Latina (Colombia, Chile, Brasil y Argentina) y de Europa (España, Alemania y Holanda) por sus experiencias innovadoras y consolidadas, se refieren a que entre los elementos más novedosos que los caracterizan están: la relación dialéctica entre la teoría y la práctica y el fomento de la investigación como una manera de reorientar la reflexión y la mejora de la docencia.

En el modelo actual de formación del docente en Cuba cobra fuerza la perspectiva que considera al docente como profesional práctico reflexivo, precisamente por la estrecha relación entre la práctica educativa, la reflexión y la investigación pedagógica.

En correspondencia con dicha relación, se reconoce la importancia de la utilización del método científico en la solución de los problemas que se presentan en la dirección del proceso pedagógico en general y en la de enseñanza-aprendizaje en particular; lo cual influye decisivamente en la elevación de la calidad de dichos procesos y en el nivel profesional del personal docente.

En la actualidad, con el perfeccionamiento continuo se ha logrado consolidar la integración e interrelación del componente investigativo con la actividad académica, laboral y extensionista en el modelo de formación del profesional de la educación. En este, la actividad laboral e investigativa de los estudiantes constituye un eje esencial y, unido a ello, el desarrollo de una formación profesional asistida de carácter individual a través de una tutoría personalizada en su concepción integral.

El proceso de formación científico-investigativo inicial, en particular con el desarrollo de las habilidades investigativas, debe garantizar la adquisición por los estudiantes de los saberes necesarios que les propicie el desempeño de la función investigativa y aplicar a la realidad cotidiana dichos conocimientos y habilidades mientras conjuga su actividad laboral e investigativa con el resto de los componentes de formación.

Es importante resaltar que las investigaciones educativas en la actualidad se caracterizan por su proyección hacia la solución de problemas, lo que constituye una exigencia de primer orden que expresa el vínculo teoría-práctica.

La formación científico-investigativa inicial en el contexto de las universidades cubanas se concibe desde el diseño curricular con un carácter interdisciplinar y profesional, 
a partir de la relación estrecha entre todos los componentes de formación y especialmente se concreta a partir del trabajo científico-investigativo que desarrolla el estudiante en la actividad laboral e investigativa.

En el modelo del profesional de las carreras pedagógicas, como representación del ideal que deben alcanzar los estudiantes, se destaca el lugar preponderante que ocupa la formación científico-investigativa al plantear como propósito entre los objetivos formativos generales la utilización del método científico para darles solución a los problemas que surjan en el contexto de actuación y por esta vía, contribuir con la construcción del conocimiento científico de la realidad educativa.

Teniendo en cuenta los elementos anteriormente descritos, el proceso de formación científico-investigativo inicial (PFCII) en este contexto es entendido como aquel en el que el estudiante se apropia del sistema de conocimientos y habilidades investigativas que le permiten utilizar el método científico para contribuir a la solución de los problemas que identifica en el proceso pedagógico en general y de enseñanza-aprendizaje en particular en los contextos de actuación profesional.

Para ello, la concepción actual del PFCII de las carreras pedagógicas contempla determinadas exigencias curriculares entre las que se encuentran:

- La realización de Trabajos de Curso como forma de culminación de determinadas asignaturas en el semestre; un Trabajo de Curso integrador en el cuarto año y el Trabajo de Diploma como forma de culminación de estudios o Examen estatal en dependencia de sus resultados académicos e investigativos.

- La proyección del trabajo científico-investigativo en correspondencia con las necesidades identificadas en el contexto de actuación de la práctica laboral e investigativa.

- La contribución de todas las disciplinas que conforman el plan de estudios y la influencia de los agentes formativos en particular al PFCII, a partir del principio interdisciplinar-profesional.

En este proceso todos los agentes formativos deben proyectar su actuación a partir del necesario vínculo de los componentes de formación. Por otra parte, involucra y compromete a los sujetos (estudiantes) en la apropiación activa de conocimientos, habilidades y valores.

Sin embargo, se resalta dentro de estos agentes formativos la figura del profesortutor a partir de la acción tutorial personalizada dirigida al PFCII y en particular a la formación y desarrollo de las habilidades investigativas básicas que garanticen eficazmente el desempeño del futuro profesional en la función investigativa.

\section{La tutoría como elemento de dirección en la formación científico-investigativa del estudiante: la acción tutorial}

El término tutoría proviene de la cultura griega, en particular de la palabra tutelae que etimológicamente significa protección. Desde este punto de vista se considera al tutor como aquella persona que cuida, protege o vela por los intereses de otra persona. 
Los estudios realizados en la bibliografía revelan que la figura del tutor aparece en el maestro de la antigua Grecia, concretamente en la mayéutica socrática. Sin embargo, la instrumentación de la tutoría en la educación universitaria aparece de manera institucional en los siglos XII y XIII, es decir de la época medieval y cobra fuerza a finales del siglo $\mathrm{XX}$ e inicios del XXI, con el desarrollo vertiginoso de las tecnologías de la información y las comunicaciones (TIC) y las mayores posibilidades de acceso a la Educación Superior a partir de la Educación a Distancia.

En la actualidad la tutoría se redimensiona y es considerada clave en los procesos universitarios tanto a nivel internacional como nacional, a partir de la necesidad de atender el desarrollo integral del estudiante en su proceso de formación profesional.

Se distinguen diversos modelos de tutoría:

- En el contexto latinoamericano se identifica: la tutoría uno a uno, tutoría de asignación y asistencia y la tutoría estratégica (ARIZA; OCAMPO, 2005).

- En las instituciones universitarias europeas: el modelo académico, modelo docente (se incluye la mentoría o tutoría entre iguales), modelo de desarrollo personal y el modelo de desarrollo profesional (RODRÍGUEZ et al., 2007).

En los diferentes ámbitos universitarios, la tutoría adopta variadas formas en su organización: individual, de grupo, técnica o asesoría académica, de la diversidad, de prácticas en empresas y la tutoría entre iguales o mentoría. Se destaca entre estas el valor que posee la tutoría uno a uno por las posibilidades que brinda al personalizar la instrucción directa e individual, la retroalimentación inmediata y correctiva que tiene el tutor del tutorado.

En el escenario actual en que se desenvuelve la enseñanza universitaria la tutoría se hace presente en los modelos de educación, con un reconocimiento de la necesidad de atender al individuo en sus diversas facetas desde las habilidades y destrezas hasta su sistema de valores, en el que el futuro profesional sea un innovador, un investigador y un agente de cambio y mejoramiento social. (ARIZA; OCAMPO, 2005; BADILLO, 2007).

En relación al término de tutoría, autores como: Lázaro y Asensi (1987), Garibay (2003), Hernández y Torres (2005), Mindiola y Venet (2009) y Ojalvo (2012) relacionan aspectos comunes en las definiciones dadas: es llevada a cabo por docentes competentes y preparados para ejercer la tutoría; su función es apoyar, asesorar, guiar, acompañar en el proceso al tutorado; la atención es personalizada; se dirige a uno o varios alumnos designados; el propósito fundamental es contribuir a la formación integral del estudiante.

La tutoría personalizada implica la atención a las necesidades y potencialidades del estudiante tutorado en su proceso formativo, de manera directa y correctiva que favorezca su aprendizaje. El profesor-tutor debe adelantarse a las necesidades del estudiante, preverlas y darle seguimiento a partir de la proyección y evaluación sistemática de acciones individuales que propicien el aprendizaje y el desarrollo de forma general del estudiante.

En este sentido el profesor-tutor desempeña roles en relación con lo docente, la investigación, lo profesional y la ayuda personal; teniendo en cuenta el papel que desempeña, las cualidades y rasgos de personalidad así como funciones y propósitos específicos de la tutoría de acuerdo con su contenido esencial. 
El modelo de tutoría que prevalece en la formación inicial del profesional de la educación en Cuba como concepción general es la tutoría integral con un carácter personalizado, dirigida a todos los componentes sustantivos en una relación uno a uno y prevé el acompañamiento al tutorado durante toda la carrera, al decir de Martínez (2008) con un carácter holístico, humanista e integrador.

Así, la tutoría en la formación inicial del profesional de la educación es entendido como un proceso de orientación, ayuda sistemática y evaluación del profesor-tutor, dirigido a la adquisición por los estudiantes de los saberes teóricos, prácticos y valorativos necesarios para el desempeño de las funciones del profesional de la Educación en los contextos de actuación, a partir de acciones individuales y personalizadas. (GONZÁLEZ MIRABAL, 2015).

De forma particular, la tutoría en la formación científico-investigativa inicial (TFCII) constituye una arista de la tutoría integral que implica tener en cuenta determinados presupuestos pedagógicos y didácticos, como proceso de enseñanza-aprendizaje profesional que debe ser proyectivo e intencionado, que garantice la atención personalizada, directa y correctiva, así como la relación de ayuda eficaz, tomando como centro las particularidades del estudiante y en función de los propósitos que se plantean en el modelo del profesional de la educación en relación con dicha formación.

Para cumplir con sus funciones el profesor-tutor debe poseer características personales tales como: el respeto, la autoestima, el optimismo, la ética, la sensibilidad, la flexibilidad, la comunicación, la empatía, la voluntad de ofrecer una práctica reflexiva, el compromiso y responsabilidad con la atención individualizada al estudiante.

El perfil del profesor-tutor de forma general se corresponde con el de buen docente, con el de ejemplo y modelo de profesional. Sin embargo, para desarrollar la tutoría en la formación científico-investigativa del estudiante se requiere además de las características antes mencionadas, que el profesor posea conocimientos, habilidades, destrezas, capacidades en correspondencia con la naturaleza de dicho proceso en particular.

La acción tutorial es entendida como la intervención estratégica, dinámica y personalizada, realizada por el profesor-tutor, que se incluye o tiene lugar de forma simultánea a otras acciones del colectivo pedagógico, con el propósito de contribuir eficazmente a la formación profesional del estudiante y por ende a su desempeño en los contextos de actuación pedagógica. (GONZÁLEZ MIRABAL, 2015).

En el contexto de la tutoría individualizada en la formación científico-investigativa inicial que se desarrolla en la Educación Superior, el profesor-tutor deberá concebir cómo ofrecer los niveles de ayuda en cada encuentro, de manera que sea específica, que responda a las necesidades e intereses del estudiante tutorado y por tanto favorezca su aprendizaje.

La relación de ayuda es fundamental para ejercer con efectividad la tutoría, la cual personaliza la orientación que requiere el estudiante y contribuye a la gestión del conocimiento por este y de forma general al proceso de aprendizaje.

La acción tutorial en este contexto constituye un proceso con carácter continuo y de seguimiento al estudiante. Por tanto, resulta imprescindible tomar como punto de partida el diagnóstico del estudiante, si se es consecuente con los aportes de Vigotski (1979), lo 
que presupone conocer la zona de desarrollo real o actual, que expresa lo que el estudiante puede hacer por sí solo sin que se le brinde ayuda.

En término de diagnóstico significa conocer sus potencialidades y necesidades lo que le permite al profesor-tutor planificar el sistema de ayuda que requiere, logrando que el estudiante transite la zona de desarrollo próximo (ZDP) hasta el nivel de desarrollo potencial.

La acción tutorial tiene como propósito esencial la atención personalizada al estudiante de manera que este desarrolle y estructure el sistema de conocimientos y las habilidades investigativas y las despliegue en la realización de su trabajo científicoinvestigativo con independencia y creatividad.

La tutoría en la formación científico-investigativa constituye un proceso de orientación, ayuda sistemática y evaluación, con carácter sistémico y de seguimiento de las necesidades y potencialidades del estudiante en su actividad científico-investigativa, que implica la realización, por el profesor-tutor designado, de acciones orientadoras, de sistematización, ejecución y retroalimentación que garanticen eficacia en la formación científico-investigativa inicial del profesional de la educación, para problematizar, describir, explicar, predecir y transformar la realidad educativa; y autotransformarse con la aplicación del método científico (GONZÁLEZ MIRABAL, 2015).

\section{Metodología}

Se procedió a la caracterización del estado actual de la acción tutorial dirigida a la formación científico-investigativa del estudiante de las carreras pedagógicas en la Universidad de Sancti Spíritus. Se recopilaron y analizaron los principales documentos legales y normativos referidos al proceso de formación científico-investigativo del estudiante de las carreras pedagógicas y las evidencias contenidas acerca del papel del tutor en dicha formación.

Además, se realizó el análisis de informes escritos de los trabajos científicoinvestigativos de los estudiantes con el propósito de establecer las principales regularidades y se aplicó cuestionarios a profesores-tutores y estudiantes.

Para la decisión muestral en el diagnóstico se procedió a la estratificación de la población de profesores-tutores y estudiantes en función del perfil de las carreras, de modo que en cada estrato estuvieran representadas las particularidades con que pudiera desarrollarse la acción tutorial en cada una de ellas.

Esto permitió encuestar a profesores que ejercen la tutoría en la formación científicoinvestigativa de los estudiantes en las diferentes carreras pedagógicas de la universidad, fundamentalmente en los años tercero, cuarto y quinto, con el propósito de caracterizar el modelo actuante en la labor tutorial.

Como resultado del análisis del diagnóstico inicial, se identificaron fortalezas relacionadas esencialmente con el nivel de preparación profesional que poseen los profesores que ejercen la tutoría en la universidad y en el caso de las debilidades, con los insuficientes presupuestos didáctico-metodológicos que sustentan los procederes para 
desarrollar la acción tutorial en la formación científico-investigativa y limitaciones que se manifiestan en la realización de este accionar.

Con el propósito de lograr una mayor precisión y concreción de los procedimientos didácticos para dirigir este proceso con efectividad, en función de las particularidades del estudiante, se propuso, como resultado científico, una metodología para la tutoría en la formación científico-investigativa inicial del profesional de la educación.

\section{Metodología para la tutoría en la formación científico-investigativa del estudiante}

La metodología para la tutoría en dicha formación está constituida estructuralmente por un aparato cognitivo y un aparato instrumental, y tiene como objetivo establecer una plataforma de procedimientos didácticos dirigidos a perfeccionar la acción tutorial en la formación científico-investigativa inicial del profesional de la educación.

Se destaca como parte componente importante de dicha metodología el encuentro de tutoría, revelándose sus exigencias didáctico-metodológicas, así como su estructura. Además, la metodología diseñada se sustenta a partir de fundamentos filosóficos, sociológicos, psicológicos, pedagógicos y didácticos que permitan su organización científica, tanto en el plano teórico como en el metodológico. Dentro de los rasgos que tipifican la metodología propuesta, se encuentran las siguientes:

1. Potencia la concepción didáctica de la acción tutorial, que tiene como centro el estudiante que aprende a investigar a partir de la reflexión sistemática de su práctica.

2. La concepción de la planificación y ejecución de la acción tutorial, así como el control y evaluación de los resultados, que tiene carácter estratégico, dinámico y flexible.

3. El diseño de la acción tutorial para la formación científico-investigativa del estudiante, que tiene como premisa esencial propiciar el desarrollo de habilidades investigativas básicas.

4. En los procedimientos se particularizan las acciones de enseñanza y de aprendizaje, en correspondencia con las exigencias didácticas que se plantean como sustento de la metodología para la tutoría en la formación científico-investigativa inicial.

5. Concibe el establecimiento de relaciones de comunicación caracterizada por el respeto, el diálogo amistoso y sincero, la confianza y la ética científica; como aspectos fundamentales en la interacción del profesor-tutor y estudiante-tutorado; así como la coordinación con el resto del colectivo pedagógico.

Las exigencias didáctico-metodológicas constituyen un sustento importante para la elaboración e implementación de la metodología que se propone:

1. Integrar de forma coherente desde la planificación de la acción tutorial hasta el control y evaluación de los resultados, la concepción que tiene en cuenta el proceso y resultado de la actividad científico-investigativa del estudiante, en particular del desarrollo que alcanza este en las habilidades investigativas. 
2. Concebir la realización de las acciones investigativas del estudiante tutorado en el marco de la actividad laboral e investigativa que desarrolla, en estrecha relación con el modo de actuación y perfil profesional.

3. Lograr el correcto aprovechamiento, desde el punto de vista didáctico, de las potencialidades del encuentro para la atención personalizada del estudiante y la potenciación de su desarrollo en las habilidades investigativas, desde la acción tutorial que desarrolla el profesor-tutor.

4. Demostrar en su accionar tutorial: rigor científico, actualidad y asequibilidad en la determinación de los contenidos y acciones investigativas a desarrollar, en correspondencia con las necesidades y posibilidades del estudiante.

5. Contextualizar (jerarquizar, seleccionar y organizar) las acciones investigativas del estudiante con un carácter flexible y dinámico.

6. Lograr un nivel de compromiso y motivación en el estudiante por la actividad científico-investigativa que realiza, a partir de la significación e importancia que tiene esta en el desempeño del profesional de la educación.

La metodología se materializa mediante las siguientes etapas: planificación de la acción tutorial para la formación científico-investigativa del estudiante, preparación y ejecución del sistema de encuentros de tutoría en la formación científico-investigativa, así como la etapa de control y evaluación de resultados.

En el diseño de la concepción de la metodología se evidencian las relaciones internas que se originan entre las exigencias y procedimientos didácticos para la atención personalizada del estudiante, el establecimiento de sus relaciones fundamentales, así como la representación de sus componentes.

A continuación se describen las etapas y los pasos lógicos para su concreción.

Etapa I: Planificación de la acción tutorial para la formación científico-investigativa del estudiante.

Esta etapa le permitirá al profesor-tutor planificar el trabajo didáctico para la dirección del proceso de formación científico-investigativa del estudiante.

\section{Pasos lógicos}

1. Creación de las condiciones objetivas y subjetivas para garantizar eficiencia en la acción tutorial dirigida a la formación científico-investigativa del estudiante.

2. Proyección del sistema de encuentros.

3. Selección de ejercicios de aprendizaje.

4. Planificación del sistema de ayuda que requiere el estudiante.

Etapa II: Preparación y ejecución del sistema de encuentros de tutoría en la formación científico-investigativa.

En esta etapa se hace énfasis en la ejecución del encuentro como forma fundamental para desarrollar la tutoría en la formación científico-investigativa del estudiante, a partir de la planificación realizada en la etapa anterior. 


\section{Pasos lógicos}

1. Acciones previas al desarrollo del encuentro.

2. Acciones iniciales de retroalimentación y corrección de resultados en el encuentro de tutoría en la formación científico-investigativa.

3. Acciones durante el desarrollo del encuentro de tutoría en la formación científicoinvestigativa del estudiante.

4. Acciones conclusivas del encuentro.

\section{Etapa III: Control y evaluación de resultados.}

La etapa de control es muy importante en la metodología pues va a permitirle al profesor-tutor constatar el grado de cumplimiento de las acciones tutoriales planificadas y de forma general de las acciones diseñadas.

\section{Pasos lógicos}

1. Retroalimentación de los resultados de las tareas investigativas que desarrolla el estudiante.

2. Valoración comparativa del nivel de desarrollo en la formación científicoinvestigativa alcanzado por el estudiante.

Con el propósito de comprobar la factibilidad y rigor cientifico de la metodología propuesta se procedió a someterla a criterio de expertos, método muy empleado en las investigaciones de corte pedagógico en la actualidad. De esta forma se pudo conocer el criterio de los expertos en relación con la: 1) adecuación de los fundamentos teóricos planteados para la metodología; 2) adecuación de las exigencias didáctico-metodológicas como sustento teórico del encuentro de tutoría; 3) adecuación de las exigencias didácticometodológicas como sustento para la elaboración e implementación de la metodología; 4) adecuación de la estructura didáctica del encuentro de tutoría; 5) adecuación de los elementos estructurales de la metodología; 6) contribución de la metodología a la sistematización de habilidades investigativas; 7) contribución de la metodología a una ayuda individualizada eficaz al estudiante y 8) aplicabilidad de la metodología para los diferentes años de estudio.

La aplicación del método criterio de expertos tuvo como resultado valoraciones positivas acerca de su factibilidad y rigor científico, ya que los expertos seleccionados evaluaron los indicadores anteriores en la categoría como muy adecuado.

Evaluación experimental de la efectividad de la metodología para la tutoría en la formación científico-investigativa del estudiante

Para la evaluación de la metodología propuesta en la práctica pedagógica se emplearon los métodos de la estadística descriptiva e inferencial. El diseño pre-experimental, con un pre-test y un post-test, permitió comprobar la efectividad de la acción tutorial en la 
formación científico-investigativa de los estudiantes con la utilización de la metodología diseñada para la tutoría en la formación científico-investigativa.

Teniendo en cuenta que se aplica un diseño experimental se decidió por la aplicación de un muestreo no probabilístico con una selección muestral de forma intencional. Se seleccionaron once profesores-tutores y once estudiantes tutorados del cuarto año de dos carreras pedagógicas, pues es en este año que se desarrolla el trabajo científico estudiantil con la implicación de las habilidades investigativas esenciales y todos los estudiantes, sin excepción, deben desarrollar su Trabajo de Curso.

La efectividad de la acción tutorial en la formación científico-investigativa inicial del profesional de la educación es entendida operacionalmente como el logro alcanzado en el proceso de la acción tutorial que se expresa en el uso adecuado de los procedimientos didácticos para la atención personalizada al estudiante sobre la base de la determinación de sus necesidades y potencialidades de aprendizaje, de los contenidos y acciones investigativas que lo favorecen y de la relación de ayuda que debe establecerse en función de esas necesidades, así como en su contribución al desarrollo y sistematización del sistema de conocimientos y habilidades investigativas en correspondencia con los objetivos establecidos en el modelo de formación inicial del profesional.

A partir de la conceptualización de la variable dependiente se determinan como dimensiones e indicadores los siguientes:

Dimensión No. 1: Atención personalizada al estudiante.

Indicadores:

1. Determinación de las principales necesidades y potencialidades de aprendizaje del estudiante en relación con su formación científico-investigativa.

2. Determinación de los contenidos y acciones investigativas que favorecen su aprendizaje.

3. Correspondencia de la relación de ayuda que se establece en función de las necesidades.

Dimensión No. 2: Desarrollo y sistematización del sistema de conocimientos y habilidades investigativas.

Indicadores:

1.Adecuación de la solución dada por los estudiantes a las tareas investigativas planteadas.

2. Grado de desarrollo de las habilidades investigativas básicas que logran los estudiantes.

Para la medición de la variable se empleó una escala ordinal de muy alto (MA), alto (A), medio (M), bajo (B) y muy bajo (MB); cuya significación cualitativa varía según el contenido del indicador. Es criterio de la autora que si después de aplicar la metodología 
en el proceso de formación inicial del profesional, al comprobar los resultados se asciende un valor en la escala ordinal, ocurre un cambio significativo.

Se hicieron corresponder los items de cada uno de los instrumentos con los indicadores y dimensiones de la variable dependiente y ello permitió que fuera evaluada utilizando la mencionada escala.

\section{Descripción y función de los instrumentos aplicados}

Para la realización del pre-experimento pedagógico se elaboraron diferentes instrumentos que posibilitaron la profundización en el análisis, tanto cuantitativo como cualitativo del estado inicial (pre-test) y final (post-test) de la variable dependiente, al comparar los resultados de los indicadores y dimensiones tanto en la constatación inicial como en la final.

Dentro de estos se encuentran la guía de observación a encuentros de tutoría (antes, durante y posterior a la aplicación de la metodología) y la guía de observación a los talleres de Trabajo Científico Estudiantil en el que se observó a cada estudiante, con el propósito de evaluar antes y después de introducir la metodología propuesta el grado de desarrollo y sistematización del sistema de conocimientos y habilidades investigativas que logran los estudiantes en la concreción de dicho trabajo.

La variable efectividad de la acción tutorial en la formación científico-investigativa, en el pre-test, se comportó en un nivel bajo (45,5\%) y en un nivel medio (54,5\%), como se muestra en la Figura 1. Estos resultados evidencian la existencia de dificultades en el proceso y en los resultados obtenidos por lo que no fue efectiva la acción tutorial, lo que se expresó en el nivel de desarrollo que mostraron los estudiantes en las habilidades investigativas.

Figura 1- Gráfico de frecuencias de categorías en el pre-test por dimensión.

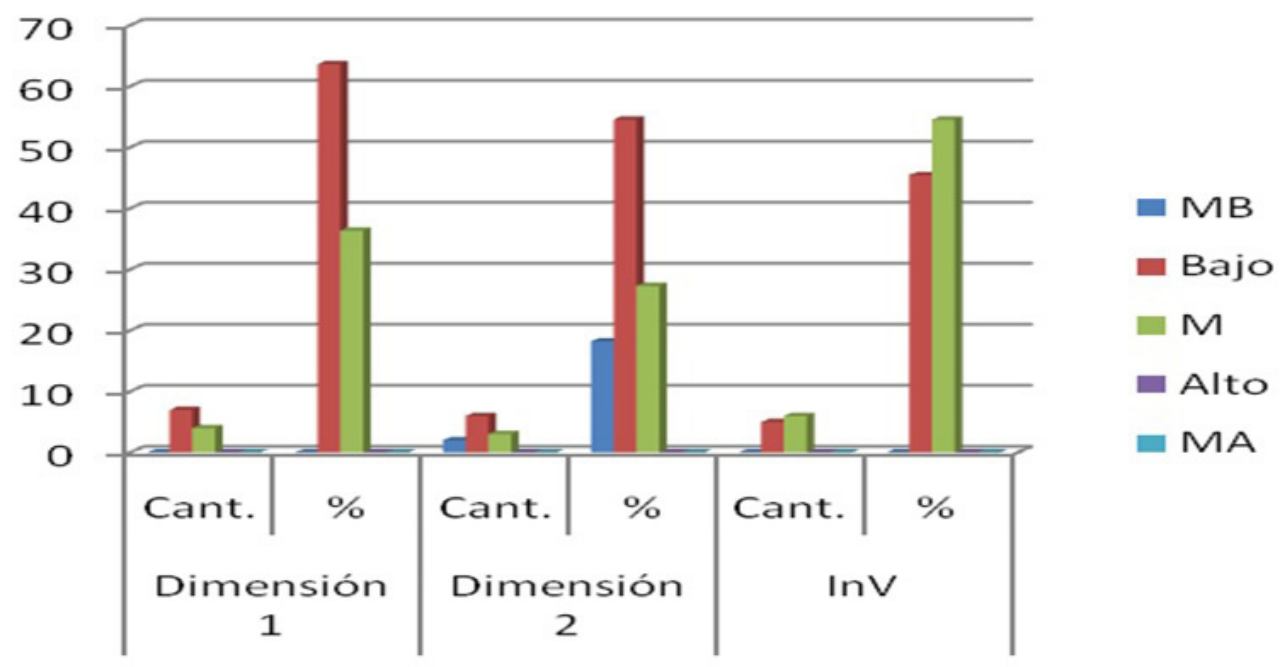




\section{Introducción de la metodología en la práctica pedagógica}

Se desarrollaron acciones de preparación para la instrumentación de la metodología las que se realizaron en los niveles organizativos para el trabajo metodológico correspondiente, con el propósito de orientar e instruir a los profesores participantes acerca de la concepción en el plano organizativo y metodológico de la metodología para su instrumentación.

Los participantes en la preparación expresaron juicios positivos en relación con la posibilidad que ofrecía la metodología para desarrollar la acción tutorial de forma más homogénea y coherente, particularmente en relación con la ejecución del encuentro de tutoría.

Durante la instrumentación de la metodología se fue haciendo un seguimiento de la evolución del estudiante, a partir de las acciones incorporadas por el profesor-tutor en cada etapa formativa, de acuerdo con el sistema de conocimientos y habilidades investigativas del año de estudio; y se corroboró en la ejecución de los talleres de trabajo científico-investigativo en el contexto de la asignatura Metodología de la investigación educativa II y III; y en los resultados a partir de la efectividad lograda en la acción tutorial desarrollada por el profesor-tutor.

En el pos-test, una vez aplicada la metodología en la práctica pedagógica, los resultados de los instrumentos de investigación evidencian, tal como se muestra en la Figura 2, que la variable efectividad de la acción tutorial en la formación científico-investigativa se comportó en un valor de 54,5\% en un nivel alto y 45,5 \% en un nivel muy alto. Estos resultados muestran la influencia de la metodología en la variable dependiente, a partir de la atención personalizada y el logro del desarrollo y sistematización de las habilidades investigativas en el estudiante y con ello la efectividad en la acción tutorial proyectada.

Figura 2- Gráfico de frecuencias de categorías en el post-test por dimensión.

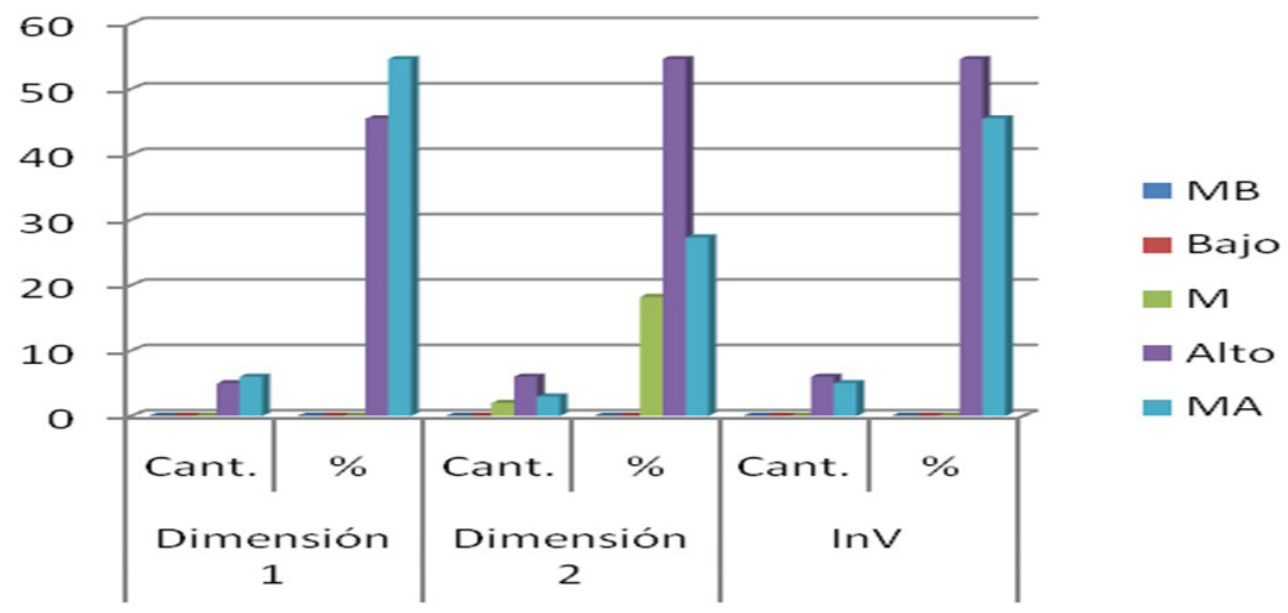


La evaluación integral de la variable dependiente, al concluir el post-test, permite afirmar que la metodología que se propone contribuye al perfeccionamiento de la tutoría en la formación científico-investigativa del estudiante, ya que con su aplicación se produjo una transformación ascendente en los indicadores de cada una de las dimensiones, con una tendencia predominante de alto y muy alto. Estos resultados, expresaron el logro alcanzado en el proceso de acción tutorial a partir de:

- El uso adecuado de los procedimientos didácticos planteados para la atención personalizada al estudiante sobre la base de: la determinación de las necesidades y potencialidades de aprendizaje del estudiante en relación con su formación científico-investigativa, de los contenidos y acciones investigativas que lo favorecen y de la relación de ayuda que debe establecerse en correspondencia.

- La contribución al desarrollo y sistematización del sistema de conocimientos y habilidades investigativas en los estudiantes tutorados, en correspondencia con los objetivos planteados en el modelo de formación inicial del profesional.

\section{Análisis estadístico de los datos con el empleo de la prueba de los rangos con signo de Wilcoxon}

La prueba estadística que se seleccionó es no paramétrica teniendo en cuenta el tipo de variable y la escala de medición empleada de carácter ordinal, con valores discretos.

Se emplea la prueba de los rangos con signo de Wilcoxon para comparar dos muestras relacionadas.

El análisis estadístico de los datos recolectados comprendió: a) la comparación de las mediciones iniciales y finales para comprobar si existen diferencias y b) inferir de los resultados que se obtengan la confirmación de la hipótesis, es decir si: $\mathrm{R}>\mathrm{M}_{1}$, donde $\mathrm{M}_{1}$ es el resultado de las mediciones iniciales (pre-test); $\mathrm{M}_{2}$ es el resultado de las mediciones finales (post-test, con la implementación de la metodología propuesta) y R es la diferencia entre las mediciones finales $\left(M_{2}\right)$ y las iniciales $\left(M_{1}\right)$.

La variable se midió con una escala ordinal y se verificaron las diferencias entre M2 y M1 determinando el valor absoluto del resultado obtenido por cada profesor-tutor y estudiante tutorado. Los valores obtenidos se convierten en rangos. Si la suma de los rangos positivos es igual a la suma de los negativos se acepta la hipótesis nula (Ho), es decir, no hay diferencias entre el pre-test y el pos-test, pero si los rangos positivos no equivalen a los negativos entonces se acepta la hipótesis alternativa (Ha): pos-test>pretest y por tanto, es efectiva la acción tutorial con la introducción de la metodología, considerando un nivel de significación $\alpha=0,05$.

Como se puede apreciar en los resultados de la prueba de Wilcoxon (Tabla 1), tomando las mediciones de profesores-tutores y estudiantes en el pre-test y post-test.

Tabla 1- Estadísticos de contraste de la Prueba de los rangos con signo de Wilcoxon.

\begin{tabular}{|c|c|}
\hline & pos - pre \\
\hline$Z$ & $-4,243$ \\
Significación asintótica (bilateral) &, 000 \\
\hline
\end{tabular}

Z- Estadístico de prueba basado en los rangos negativos

Fuente: Elaborado para este estudio. 
El valor tipificado del estadístico de prueba $(Z)$ que corresponde a la menor de las dos sumas de rangos, es igual a $-4,423$; por tanto dicho valor es estadísticamente significativo, y se rechaza la hipótesis nula, de acuerdo a que la significación asintótica es menor que el nivel de significación, lo que demuestra la influencia de la metodología propuesta en la variable dependiente: efectividad de la acción tutorial en la formación científico-investigativa inicial del profesional de la Educación.

\section{Conclusiones}

La formación científico-investigativa constituye un elemento esencial para el profesional de la Educación en el sistema educativo cubano dado su papel en el perfeccionamiento del proceso pedagógico en general y en el auto perfeccionamiento de su profesionalidad. Es por ello que ha estado presente como una arista fundamental en los planes de estudio correspondientes a la formación inicial de dicho profesional, desde su inserción en los primeros años hasta su consolidación dentro de los componentes curriculares. En este contexto, la figura del profesor-tutor ha constituido siempre un componente fundamental.

La tutoría en la formación científico-investigativa inicial desempeña un importante papel por cuanto mediante la atención personalizada al estudiante contribuye de manera significativa a la formación científico-investigativa.

La acción tutorial implica considerar una planificación didáctica que toma como punto de partida las particularidades del estudiante y una proyección estratégica de las acciones sobre la base de una orientación personalizada que implique las relaciones de ayuda adecuadas en correspondencia con el nivel de desarrollo del estudiante y las exigencias de su etapa de formación.

La metodología para la tutoría en la formación científico-investigativa inicial del profesional de la educación supone la realización de procedimientos didácticos secuenciados de manera lógica y coherente en sus etapas de planificación, de ejecución y de control y evaluación. Dichos procedimientos posibilitan al profesor-tutor conducir la formación del estudiante a través de una relación de ayuda que orienta su desarrollo y apoya sus necesidades en la actividad científico-investigativa de forma que contribuye a la sistematización de sus habilidades en esta dirección y de forma general a su formación científico-investigativa.

La metodología que se propone fue sometida a una evaluación mediante Criterio de expertos, quienes evaluaron positivamente su factibilidad y rigor científico. Los resultados obtenidos en la evaluación de su aplicación en la práctica pedagógica mediante un preexperimento aplicado con una muestra seleccionada de profesores-tutores y estudiantes permitió constatar cambios favorables significativos en el proceso tutorial y en los resultados que alcanzan los estudiantes en el desarrollo de sus habilidades investigativas y en su formación científico-investigativa de forma general, lo que evidencia que se logra efectividad en la acción tutorial con la aplicación de la metodología propuesta. 


\section{Referencias}

ARIZA, Gladys Ibeth; OCAMPO, Héctor Balmes. El acompañamiento tutorial como estrategia de la formación personal y profesional: un estudio basado en la experiencia en una institución de educación superior. Universitas Psychologica, Bogotá, v. 4, n. 1, p. 31-41, 2005. Disponible en: <http://redalyc.uaemex.mx/ pdf/647/64740104.pdf>. Acceso en: 26 oct. 2012.

BADILLO, Jessica. La tutoría como estrategia viable de mejoramiento de la calidad de la educación superior. Reflexiones en torno al curso. Revista de Investigación Educativa, Veracruz, n. 5, p. 1-22, jul./dic. 2007. Disponible en: <http://www.uv.mx/cpue/num5/practica/completos/badillo_tutoria.pdf>. Acceso en: 26 oct. 2012.

CASTELLANOS, Beatriz. Investigación educativa: nuevos escenarios, nuevos actores, nuevas estrategias. La Habana: Instituto Superior Pedagógico "Enrique José Varona", 1998.

CHIRINO, María Victoria. Perfeccionamiento de la formación inicial investigativa de los profesionales de la educación. La Habana: Instituto Superior Pedagógico "Enrique José Varona", 2002. 212 p. Tesis (Doctorado en Ciencias Pedagógicas) - Instituto Superior Pedagógico “Enrique José Varona”, La Habana, 2002.

FERNÁNDEZ MUÑOZ, Ricardo. El cambio educativo desde la profesionalización del docente: hacia la configuración de un modelo de profesor investigador. Docencia e Investigación, Toledo, v. 20, n. 5, p. 57-70, 1995.

GARCÍA BATISTA, Gilberto; ADDINE FERNÁNDEZ, Fátima. La formación investigativa del docente. Un reto del nuevo milenio. In: GARCÍA BATISTA, Gilberto. (Coord.). Profesionalidad y práctica pedagógica. La Habana: Pueblo y Educación, 2004. p. 71- 76.

GARIBAY, Gabriela. Programa Institucional de Tutorías. Guadalajara: [s. n.], 2003. Disponible en: <http:// campusdigital.uag.mx/>. Acceso en: 12 oct. 2013.

GONZÁLEZ MIRABAL, Diane. La tutoría en la formación científico-investigativa inicial del profesional de la educación. 2015. 210 p. Tesis (Doctorado en Ciencias Pedagógicas) - Universidad Sancti Spíritus, La Habana, 2015.

HERNÁNDEZ, Vicente; TORRES, Jorge. Informe técnico: la acción tutorial en la universidad. Madrid: Universidad Pontificia Comillas de Madrid, 2005. Disponible en: <http://www.uah.es/empresariales/ estudiantes/tutorias/Plan_Acc_Tutorial.pdf>. Acceso en: 15 febr. 2013.

LAGUNA, Jorge Alejandro; SÁNCHEZ, Alba. El maestro investigador: alternativas didácticas para su preparación inicial. 2005. Disponible en: <http://www.monografias.com/trabajos30/>. Acceso en: 19 mzo. 2013.

LÁZARO, Ángel; ASENSI, Jesús. Manual de orientación escolar y tutoría. Madrid: Nancea, 1987. Disponible en: <http://www.iberlibro.com/>. Acceso en: 3 jun. 2013. 
LÓPEZ, Lutgarda. El desarrollo de habilidades de investigación en la formación inicial de profesorado de química. 2001. 188 p. Tesis (Doctorado en Ciencias Pedagógicas) - Instituto Superior Pedagógico "Conrado Benítez", Cienfuegos, 2001.

LÓPEZ, Lutgarda, PÉREZ, Coralia; CÁCERES, Maritza. Maestro investigador: un reto en la formación del profesorado de ciencias. Pedagogía Universitaria, La Habana, v. 9, n. 3, p. 105-114, 2004. Disponible en: <http://cvi.mes.edu.cu/peduniv/base-1/2004-vol.-ix-no.-3/>. Acceso en: 19 mzo. 2013.

MARTíNEZ, Mercedes. El tutor pedagógico en la formación docente. Varona, La Habana, n. 46, p. 19-26. 2008. Disponible en: <http://www.varona.rimed.cu/revista_varona/index.php?>. Acceso en: 30 nov. 2012

MINDIOLA, Eudis; VENET, Regina. Competencias tutorales: una propuesta de formación en el maestro tutor del PNFE de Misión Sucre. 2009. Disponible en: <http://www.monografias.com/trabajos90/>. Acceso en: 23 febr. 2014.

OJALVO, Victoria. Orientación y tutoría como estrategia para elevar la calidad de la educación. In: CONGRESO INTERNACIONAL UNIVERSIDAD. Curso Universidad 2012, La Habana, Actas... La Habana: Ministerio de Educación Superior, 2012. 1 CD-ROM.

OREALC. Oficina Regional de Educación para América Latina y El Caribe; UNESCO. Organización para la Educación, la Ciencia y la Cultura de las Naciones Unidas. Modelos innovadores en la formación inicial docente. Santiago de Chile: Andros, 2006.

PÉREZ, Gastón. Fundamentos que deben regir la formación investigativa de los maestros. 2005. Disponible en: <http://www.monografias.com/trabajos28/formacion-investigativa-maestro/formacion-investigativamaestro.shtml>. Acceso en: 6 dic. 2012.

PORLÁN, Rafael. El maestro como investigador en el aula: investigar para conocer, conocer para enseñar. Investigación en la Escuela, Sevilla, n. 1, p. 63-69, 1987. Disponible en: <http://www. investigacionenlaescuela.es/articulos/1/R1_9.pdf>. Acceso en: 23 oct. 2012.

RODRÍGUEZ, María Victoria et al. La acción tutorial en la universidad en el marco del Espacio Europeo de Educación Superior EEES. In: JORNADAS DE ASEPUMA, 15. y ENCUENTRO INTERNACIONAL, 3. Oviedo, 2007. Actas... Oviedo: Universidad de Oviedo, 2007. Disponible en: <http://www.uv.es/asepuma/XV/ comunica/606.pdf>. Acceso en: 24 oct. 2012. p. 1-12.

SALAZAR, Diana. Cultura científica y formación interdisciplinaria de los profesores en la actividad científico investigativa. In: ADDINE, Fátima (Coord.). Didáctica: teoría y práctica. La Habana: Pueblo y Educación, 2004. p. 234-250.

STENHOUSEN, Lawrence. La investigación como base de la enseñanza. 4. ed. Madrid: Morata, 1987.

TONUCCI, Francesco. La escuela como investigación. Buenos Aires: Miño y Dávila, 1988.

UNESCO. Organización para la Educación, la Ciencia y la Cultura de las Naciones Unidas. Declaración 
Mundial sobre la Educación Superior en el siglo XXI: visión y acción. In: CONFERENCIA MUNDIAL SOBRE LA EDUCACIÓN SUPERIOR, Paris, 1998. Informe final. Paris: Unesco, 1998. p. 19-30. Disponible en: <http:// www.unesco.org/education/educprog/wche/declaration_spa.htm>. Acceso en: 02 mayo 2013.

VIGOTSKY, Lev. El desarrollo de los procesos psicológicos superiores. Barcelona: Crítica, 1979.

Recebido em: 09.09.2016

Modificações em: 22.11.2016

Aprovado em: 04.04.2017

Diane González Mirabal es Doctora en Ciencias Pedagógicas, profesora titular en la Universidad de Sancti Spíritus, Cuba.

Gustavo Esteban Achiong Caballero es Doctor en Ciencias Pedagógicas, profesor titular y de mérito de la Universidad de Sancti Spíritus "José Martí Pérez", Cuba. 